DOI: https://doi.org/10.46296/yc.v6i10edespfeb.0151

\title{
DETERMINACIÓN SIMULTÁNEA DE ANTINFLAMATORIOS NO ESTEROIDEOS EN AGUA PARA CONSUMO HUMANO MEDIANTE HPLC- DAD
}

\section{SIMULTANEOUS DETERMINATION OF NON-STEROIDAL ANTI- INFLAMMATORIES IN WATER FOR HUMAN CONSUMPTION BY HPLC- DAD}

\author{
Franco-Zambrano Byron Fernando 1; Bravo-Sánchez Luis Ramón 2; \\ Ruiz-Reyes Enrique ${ }^{3}$ \\ ${ }^{1}$ Ingeniero en Recursos Naturales y Ambiente, Maestrante del Instituto de Postgrado de la \\ Universidad Técnica de Manabí, UTM. Portoviejo, Ecuador. Correo: bfranco0869@utm.edu.ec. \\ ORCID ID: https://orcid.org/0000-0003-3421-9388. \\ 2 Doctor en Ciencias Químicas, Departamento de Ciencias de la Tierra, Universidad Estatal \\ Amazónica (UEA), Vía Tena Km $2{ }^{1 / 2}$. Puyo, Ecuador. Correo: Ibravo@uea.edu.ec. \\ ${ }^{3}$ Doctor en Ciencias Químicas, Departamento de Química, Instituto de Ciencias Básicas, \\ Universidad Técnica de Manabí. Portoviejo, Ecuador. Correo: enrique.ruiz@utm.edu.ec.
}

\section{Resumen}

El crecimiento de la población urbana presenta una serie de retos técnicos, ecológicos y sociales para asegurar la disponibilidad y calidad de las aguas superficiales por la presencia de contaminantes emergentes, los mismos que en los últimos años han despertado un interés científico por su creciente presencia en medios acuosos. El objetivo de este estudio fue la determinación simultánea de seis antinflamatorios no esteroideos: aspirina (ácido acetil salicílico), diclofenaco, ibuprofeno, ketorolaco, meloxicam y naproxeno, mediante cromatografía líquida de alta eficacia con un detector ultravioleta - visible multiespectral de arreglo de diodos (HPLC-DAD). Se establecieron diferentes puntos de muestreo del agua a lo largo del río Portoviejo, cercanos a zonas de captación de agua para consumo, así como también se recolectaron muestras de agua potable en las ciudades de Manta, Portoviejo y Rocafuerte. Los primeros resultados revelaron que las concentraciones de estos contaminantes se encontraron por debajo de los límites de detección y cuantificación de la técnica de análisis, por lo que no fue posible su cuantificación en niveles por debajo de los $\mu \mathrm{g} / \mathrm{mL}$. Los resultados sugieren que para la evaluación de estos contaminantes se necesita de técnicas analíticas más sensibles para determinar concentraciones de estos fármacos a niveles de $\mathrm{ng} / \mathrm{L}$.

Palabras claves: Contaminantes emergentes; antinflamatorios no esteroideos; cromatografía líquida de alta eficacia, detector UV-vis multiespectral.

\begin{abstract}
The growth of the urban population triggers a series of technical, ecological and social challenges to ensure the availability and quality of surface water due to the presence of emerging contaminants, which in recent years have aroused scientific interest due to their increasing presence in aqueous media. The objective of this study was the simultaneous determination of six non-steroidal anti-inflammatory drugs (aspirin, diclofenac, ibuprofen, keterolac, meloxicam and naproxen) by high performance liquid chromatography coupled to a diode array detector (HPLC-DAD). Different water sampling points were established along the Portoviejo River near drinking water catchment areas, as well as drinking water samples were collected in the cities of Manta, Portoviejo and Rocafuerte. Initial results revealed that the concentrations of these contaminants were found to be below the analytical technique detection and quantification limits, making impossible to quantify them at $\mu \mathrm{g} / \mathrm{L}$ levels, both in surface water and drinking water
\end{abstract}

Información del manuscrito:

Fecha de recepción: 26 de noviembre de 2021.

Fecha de aceptación: 27 de enero de 2022.

Fecha de publicación: 15 de febrero de 2022. 
samples. The results also suggest that more sensitive and accurate analytical techniques are needed for the evaluation of these contaminants to determine concentrations of these drugs at ng/L levels.

Keywords: Emerging pollutants; non-steroidal anti-inflammatory drugs; high performance liquid chromatography, diode array detector.

\section{Introducción}

La población urbana del mundo ha crecido rapidamente en los ultimos años. Según Jensen \& Wu (2018), el $54 \%$ de la población mundial vive en zonas urbanas y se prevé que esta proporción aumente al $66 \%$ para el año 2050. El crecimiento de la población urbana presenta una serie de retos técnicos, ecológicos y sociales por ser un factor significativo que afecta la disponibilidad y calidad de las aguas superficiales y subterráneas por la presencia de contaminantes persistentes (Larsen \& Gujer, 1997; Pal et al., 2014).

Tradicionalmente el agua dulce se ha extraído de cuerpos de aguas superficiales y subterráneos locales o se ha importado de cuencas hidrográficas lejanas, sin embargo con la creciente demanda de recursos hídricos por parte de las megaciudades, las instituciones competentes de la gestión hídrica se han visto obligadas a desarrollar nuevos suministros de agua a partir de fuentes deterioradas, como pueden ser ríos o lagos que reciben descargas de efluentes residuales, escorrentía de aguas pluviales, etc. Este ciclo de procesos de captación, potabilización, distribución y saneamiento se conoce como Ciclo Urbano del Agua o UWC (Urban Water Cycle, por sus siglas en inglés) (Jekel et al., 2013; Marsalek, 2014).

De acuerdo a Geissen et al. (2015), el flujo de contaminantes presente en el UWC incluye ciertos productos químicos sintéticos o naturales que no se vigilan comúnmente pero que tienen el potencial de causar efectos adversos en los ecosistemas acúaticos y en la salud humana, a estos se los denomina contaminantes emergentes (CEs); los cuales incluyen productos farmaceúticos, drogas ilegales o de 
abuso, productos de cuidado personal, disruptores endócrinos, retardantes de fuego, etc. (Farré et al., 2008).

Las investigaciones sobre estos compuestos han revelado su creciente presencia en los cuerpos de agua superficiales, por ser resistentes a la biodegradación y altamente persistentes en el medio acuoso; resultando en una exposición crónica capaz de producir efectos ecotoxicológicos como alteraciones en la reproducción y feminización en peces (Fatta et al., 2007; Rivera et al., 2013; Ruhí et al., 2016).

Los antiinflamatorios no esteroideos (AINEs) son uno de los grupos de fármacos más accesibles y prescritos a nivel mundial, son útiles en el dolor reúmatico, tanto en enfermedades inflamatorias como degenerativas por su poder analgésico (Rosas et al., 2013). De acuerdo a Ortiz et al. (2014) según datos del Banco Central del Ecuador el ibuprofeno, la aspirina, el diclofenaco y el naproxeno se encuentran entre la lista de los 10 medicamentos más comercializados sin receta médica en el país durante el período 2011-2012.

Los productos farmacéuticos pueden ingresar en al ambiente como productos originales 0 metabolitos, principalmente a través de la excreción en la orina y su posterior eliminación en el sistema hidrosanitario (Ebele et al., 2017). La principal fuente de contaminación puntual de compuestos farmacéuticos en cuerpos de agua son los efluentes procedentes de hospitales, clínicas y centros médicos, con contribuciones de efluentes residuales industriales; así mismo la lixiviación en campos agrícolas por las prácticas de ganadería intensiva aportan un flujo considerable de fármacos de uso veterinario (Houtman, 2010; Lin et al., 2010) (figura 1). 
Figura 1. Fuentes y destino de los productos farmacéuticos en el ambiente.

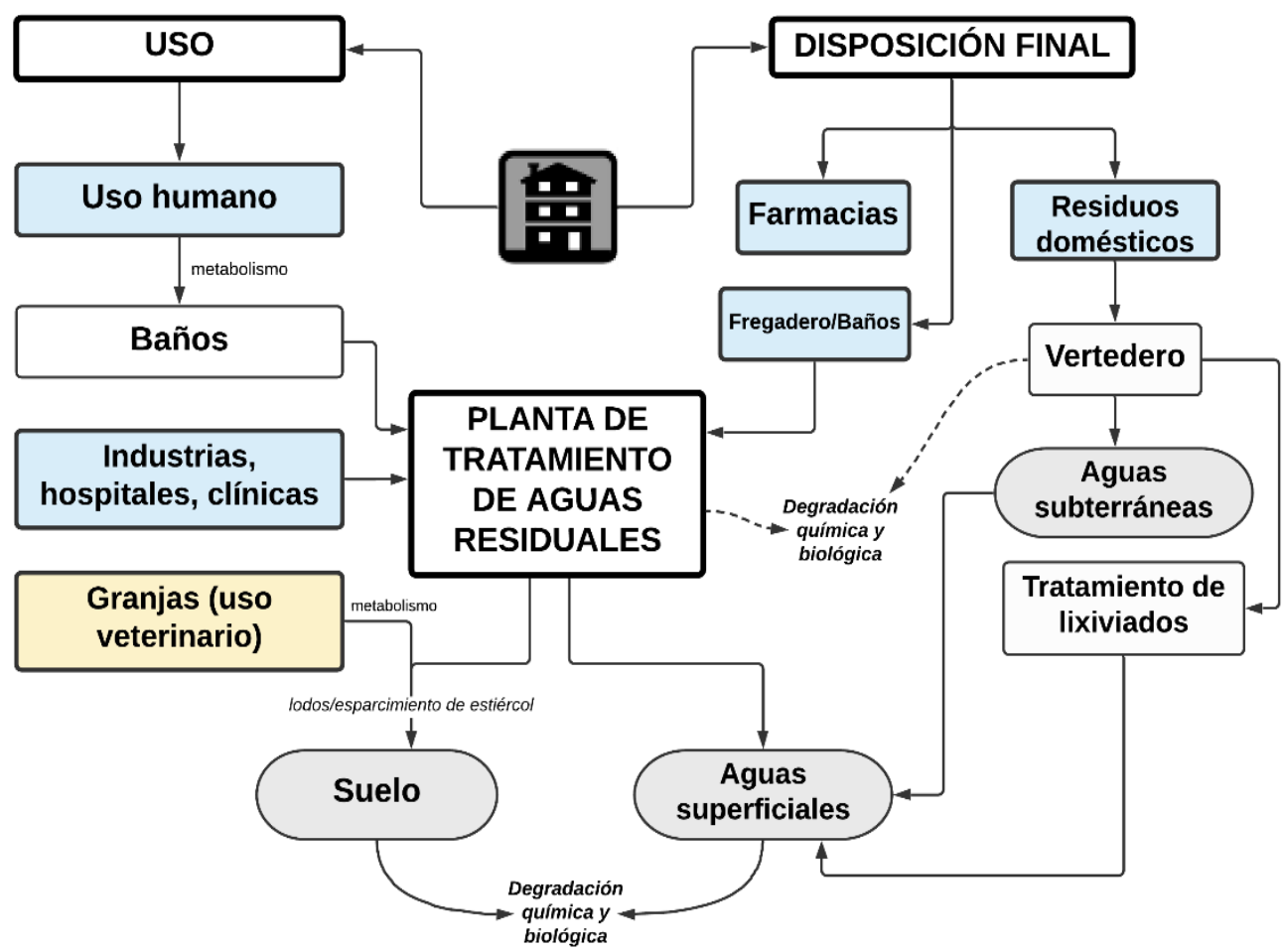

Fuente: Adaptado de "Occurrence patterns of pharmaceuticals in water and wastewater environments" (p. 1227), por Nikolaou A., Meric S., Fatta D., 2007. Analytical and Bioanalytical Chemistry.

Según Jardim et al. (2012) la mayoría de procesos de las plantas convencionales de tratamiento de aguas residuales no están diseñados para eliminar totalmente estos contaminantes, lo que supone un desafío para el control y la gestión del ciclo urbano del agua, resultando imprescindible para asegurar estándares de calidad en el agua para consumo humano.

La investigación científica sobre los contaminantes emergentes se ha ampliado a escala mundial, paralelamente, se están dedicando grandes esfuerzos a la parte analítica, con el fin de desarrollar y validar métodos suficientemente sensibles y específicos para la identificación y cuantificación de estos contaminantes (Fatta et al., 2011; Kostopoulou \& Nikolaou, 2008; Peña et al., 2019).

El objetivo de este estudio es determinar seis fármacos a través de una técnica analítica para su separación simultánea en muestras acuosas bajo las condiciones cromatográficas adecuadas para su cuantificación. 


\section{Materiales y métodos}

\section{Área de estudio}

Se seleccionaron seis puntos de muestreo a lo largo del del río Portoviejo, el criterio de selección fue la cercanía a zonas de captación de agua para consumo.

El primer punto de muestreo (P1) estaba situado cerca de la estación de bombeo "Caza Lagarto", que abastece de agua cruda a ciudades como Manta. El punto de muestreo (P2) está situado en el río Portoviejo, entre los límites territoriales del cantón Santa Ana y Portoviejo, sector Estancia Vieja. El punto de muestreo (P3) está situado cerca de la estación de captación de la planta potabilizadora de la ciudad de Portoviejo. El cuarto punto de muestreo (P4) se encuentra en el río
Portoviejo, sector Picoazá, se sabe que las aguas de este punto son de mala calidad puesto que reciben aguas residuales sin tratar de las zonas urbanas adyacentes. El punto de muestreo (P5) y (P6) están situados en el río Portoviejo, sector Mejía y Ceibal, respectivamente, las aguas superficiales de estos puntos reciben vertidos crudos $\mathrm{y}$ tratados de las regiones urbanas vecinas y se utilizan para abastecer parcialmente de agua a la ciudad de Manta (figura 2).

En las ciudades de Manta, Portoviejo y Rocafuerte, también se establecieron sitios de muestreo de agua potable. En este caso, las muestras se recogieron directamente de los grifos conectados a la red de distribución principal de cada ciudad.

Figura 2. Ubicación de los puntos de muestreo a lo largo de la cuenca hidrográfica del río Portoviejo.

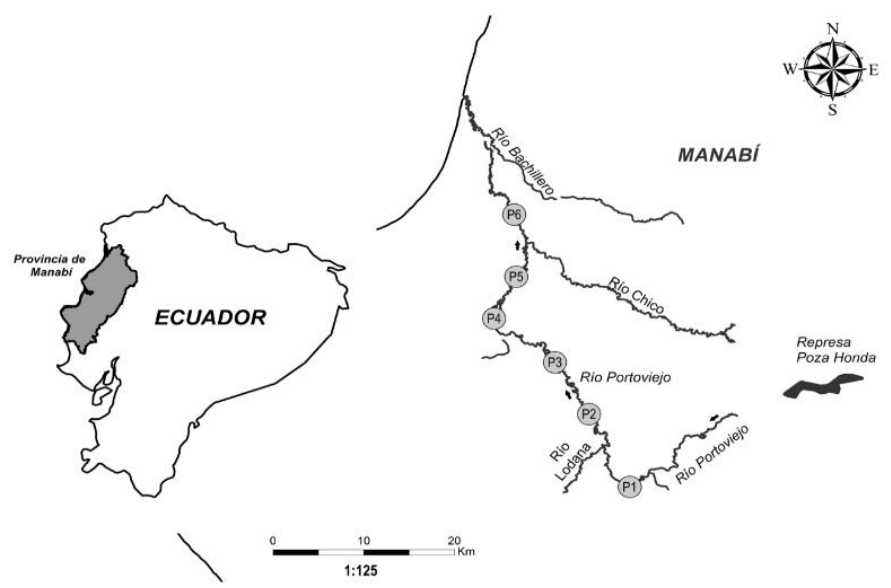

Fuente: Elaboración propia. 


\section{Materiales y reactivos}

Se determinaron seis fármacos en las muestras de agua: Aspirina (ASP), Diclofenaco (DCF), Ibuprofeno (IBF), Keterolaco (KTL), Meloxicam (MLX) y Naproxeno (NPX), todos estos estándares analíticos se obtuvieron de SigmaAldrich® (St. Louis, EE.UU.). Las estructuras químicas de los compuestos investigados se presentan en la figura 3.

Todos los disolventes y reactivos empleados durante el analísis químico eran de alta pureza. El acetonitrilo y el agua ultra pura grado HPLC, se obtuvieron de la marca Merck®, además se utilizó ácido acético e hidróxido de amonio al 25\% de grado analítico.

\section{Instrumentos y equipos}

Los compuestos analizados se determinaron mediante cromatografía líquida de alta eficacia acoplado a un detector de arreglo de diodos (sistema Agilent ${ }^{\circledR}$ HP 1100 con detector de diodos Agilent ${ }^{\circledR}$ 1100 series) según la literatura consultada (Patel et al., 2013). En resumen, la separación cromatográfica se realizó en una columna Hypersil Gold aQ (150 x 4,6 mm, $5 \mu \mathrm{m}$ Thermo Scientific ${ }^{\mathrm{TM}}$ ) a 35 ${ }^{\circ} \mathrm{C}$, la fase móvil usada consistió en dos mezclas de solventes; la mezcla A estuvo constituida por acetonitrilo con $2 \%$ de ácido acético y la mezcla $\mathrm{B}$ por buffer de acetato de amonio $\mathrm{pH}$ 4,2 con $10 \%$ de acetonitrilo y $2 \%$ de ácido acético; se ejecutó un gradiente elución a una velocidad de flujo de 0,9 $\mathrm{mL} / \mathrm{min}$. El programa del gradiente se muestra en la tabla 1. La detección múltiple de los componentes se realizó a $230 \mathrm{~nm}$ (ASP, IBF, NPX), 254 nm (DCF, $\mathrm{KTL})$ y $360 \mathrm{~nm}(\mathrm{MLX})$.

Figura 3. Estructura química de los fármacos estudiados.

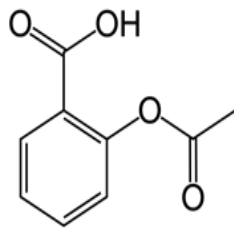

Aspirina

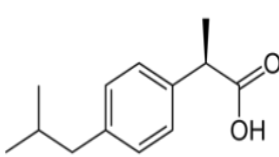

Ibuprofeno

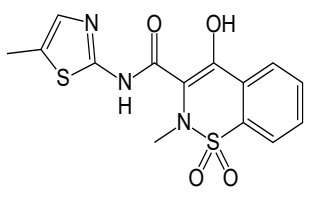

Meloxicam

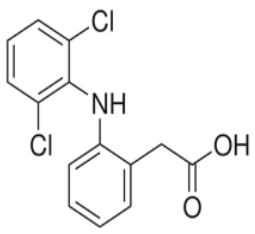

Diclofenaco<smiles>O=C(c1ccccc1)c1ccc2n1CCC2C(=O)O</smiles>

Keterolaco

Naproxeno<smiles>COc1ccc2cc([C@@H](C)C(=O)O)ccc2c1</smiles>

Fuente: Elaboración propia. 
Tabla 1. Programa de elución de gradiente.

\begin{tabular}{ccccc}
\hline & Tiempo $(\boldsymbol{m i n})$ & Flujo $(\mathbf{m L} / \mathbf{m i n})$ & \%A & \%B \\
\hline 1 & & 0,9 & 35,0 & 65,0 \\
2 & 3,0 & 0,9 & 35,0 & 65,0 \\
3 & 16,0 & 0,9 & 55,0 & 45,0 \\
4 & 20,0 & 0,9 & 75,0 & 25,0 \\
5 & 25,0 & 0,9 & 75,0 & 25,0 \\
6 & 30,0 & 0,9 & 35,0 & 65,0 \\
\hline
\end{tabular}

Fuente: Elaboración propia.

\section{Muestreo y preparación de las muestras}

Las muestras de agua superficial y potable se recogieron utilizando botellas de vidrio ámbar de $1 \mathrm{~L}$ previamente limpiadas con agua destilada. La extracción en fase sólida de las aguas superficiales y potables se llevó a cabo utilizando cartuchos de Octadecilsilano Supelclean LC-18, 1g (6 mL), con la finalidad de concentrar entre 100 a 200 veces cada muestra, luego se pasaron por filtros de jeringa de 17 $\mathrm{mm}$ de nylon de $0,45 \mu \mathrm{m}$ de tamaño de poro; previo a su inyección en el sistema de HPLC.

El acondicionamiento de los cartuchos y la elución de los analitos se realizó con acetonitrilo, tal y como se describe en otros métodos previamente validados (Leiva, 2019; Patrolecco et al., 2013).

\section{Resultados y discusión}

\section{Calibración}

La disolución madre se preparó en un matraz aforado de $100 \mathrm{~mL}$ disolviendo $75 \mathrm{mg}$ de cada fármaco (NPX, ASP, DCF, KTL, MLX, IBF) en $30 \mathrm{~mL}$ de agua grado HPLC y $70 \mathrm{~mL}$ de acetonitrilo, posteriormente se pasaron alícuotas de $1 \mathrm{~mL}$ de disolución madre a matraces de 10 $\mathrm{mL}$ y se enrasaron con agua/acetonitrilo $(30: 70 \mathrm{v} / \mathrm{v})$, se repitió el proceso hasta obtener seis niveles de concentración de cada fármaco y se procedió a realizar las tablas y las curvas de calibración (Singh, K.B., Waikar, S.B., and Padmane, S.P., 2012). En la figura 4 se muestra el cromatograma típico de una disolución mixta con los estándares en estudio. 
Figura 4. Cromatograma típico de la solución mixta con los estándares en estudio.

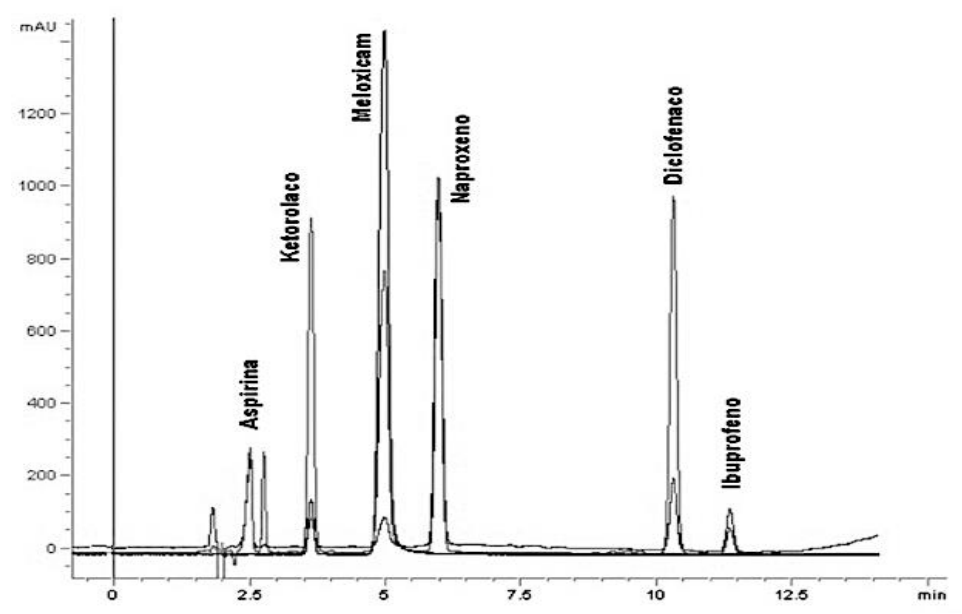

Fuente: Elaboración propia.

\section{Validación del método}

Se realizaron experimentos para determinar los parámetros experimentales que afectan a la separación cromatográfica de todos los fármacos. Los experimentos iniciales mostraron una mejor resolución y forma de los picos utilizando acetonitrilo como modificador orgánico para el desarrollo del método, en comparación con el metanol; así lo indicaba Patel et al. (2013) en la literatura.

Se probaron los efectos de la composición del acetato de amonio y el fosfato como reguladores de $\mathrm{pH}$ en la fase móvil con vistas a mejorar la resolución y simetría de los picos. Finalmente se utilizó el buffer de acetato de amonio de $\mathrm{pH} 4,2$, con el cual se logró la mejor resolución para todos los analitos en un tiempo de ejecución de 30 min.

Los límites de detección (LOD) y límites de cuantificación (LOQ) se determinaron a través de la relación señal/ruido de los analitos, el valor más bajo de límite de detección fue de $0,15 \mu \mathrm{g} / \mathrm{mL}$ mientras que el de cuantificación más alto fue de 6,52 $\mu \mathrm{g} / \mathrm{mL}$ respectivamente. Los resultados se muestran en la tabla 2 .

Tabla 2. $L O D$ y $L O Q$ de los fármacos estudiados.

\begin{tabular}{lllllll}
\hline Fármaco $(\mu \mathrm{g} / \mathrm{mL})$ & ASP & KTL & MLX & NPX & DCF & IBF \\
\hline LOD & 0,75 & 0,15 & 0,21 & 0,15 & 0,32 & 2,41 \\
LOQ & 2,31 & 0,62 & 0,74 & 0,58 & 1,10 & 6,52 \\
\hline
\end{tabular}

Fuente: Elaboración propia. 
Para la evaluación de la linealidad se prepararon disoluciones estándares en el intervalo de concentraciones de 3,75 a $75,0 \mu \mathrm{g} / \mathrm{mL}$ para ASP, KTL, NPX, DCF, IBF y de 2,62 a 52,5 $\mu \mathrm{g} / \mathrm{mL}$ para MLX, con el fin de obtener seis niveles de concentración para cada fármaco. Las curvas de calibración se obtuvieron por regresión lineal de las áreas de los picos frente a su concentración respectiva (A. Nikolaou, 2013; Nikolaou et al., 2007). La prueba de linealidad se realizó con la mezcla estándar a diferentes concentraciones, donde el coeficiente de correlación varió en un intervalo estrecho desde $\mathrm{R}=$ 0,9900 para el keterolaco hasta $R=$ 0,9999 para la aspirina; en la tabla 3 se resumen los resultados.

La repetibilidad se determinó inyectando cinco réplicas de cada disolución estándar que cubrieran niveles de concentración de bajo a alto, todo esto en un mismo día. Los valores de desviación estándar relativa de la repetibilidad del método estuvieron por debajo del $20 \%$ (tabla 4). Con los resultados, se demostró que el método desarrollado es preciso.

Tabla 3. Resultados de linealidad.

\begin{tabular}{lcccc}
\hline Fármacos & $\begin{array}{c}\text { Intervalo } \\
(\boldsymbol{\mu} \mathbf{g} / \mathbf{m L})\end{array}$ & $\begin{array}{c}\text { Longitud de } \\
\text { Onda }(\mathbf{n m})\end{array}$ & $\begin{array}{c}\text { Ecuación de } \\
\text { regresión }\end{array}$ & $\begin{array}{c}\text { Coeficiente de } \\
\text { Correlación } \\
(\boldsymbol{R})\end{array}$ \\
\hline ASP & $3,75-75$ & 230 & $y=22,053 x+2,37$ & 0,99995 \\
KTL & $3,75-75$ & 254 & $y=9,019 x+30,64$ & 0,99009 \\
MLX & $2,62-52,5$ & 360 & $y=49,666 x-92,13$ & 0,99121 \\
NPX & $3,75-75$ & 230 & $y=130,238 x+151,05$ & 0,99954 \\
DCF & $3,75-75$ & 230 & $y=23,965 x-12,96$ & 0,99799 \\
IBF & $3,75-75$ & 254 & $y=0,864 x+0,380$ & 0,99980 \\
\hline
\end{tabular}

Fuente: Elaboración propia.

Tabla 4. Resultados de repetibilidad.

\begin{tabular}{lccc}
\hline Fármacos & $\begin{array}{c}\text { Concentración } \\
(\boldsymbol{\mu g} / \mathbf{m L})\end{array}$ & Media \pm SD $(\boldsymbol{\mu g} / \mathbf{m L})$ & RSD (\%) \\
\hline \multirow{2}{*}{ ASP } & 3 & $3,90 \pm 0,4$ & 10,26 \\
& 73 & $73,78 \pm 2,15$ & 2,92 \\
\hline \multirow{2}{*}{ KTL } & 4 & $4,18 \pm 0,53$ & 12,79 \\
& 92 & $92,07 \pm 7,41$ & 8,05 \\
\multirow{2}{*}{ MLX } & 2 & $2,58 \pm 0,27$ & 10,80 \\
& 39 & $39,54 \pm 1,86$ & 4,71 \\
\hline
\end{tabular}




\begin{tabular}{lccc}
\hline \multirow{2}{*}{ NPX } & 4 & $4,04 \pm 0,33$ & 8,22 \\
& 64 & $64,09 \pm 2,08$ & 3,21 \\
\hline \multirow{2}{*}{ DCF } & 3 & $3,64 \pm 0,47$ & 13,07 \\
& 67 & $67,10 \pm 1,94$ & 2,90 \\
\hline \multirow{2}{*}{ IBF } & 3 & $3,68 \pm 0,41$ & 11,32 \\
& 62 & $62,62 \pm 1,78$ & 2,85 \\
\hline
\end{tabular}

Fuente: Elaboración propia.

\section{Muestras de agua superficial}

Las concentraciones de los compuestos investigados en las muestras de aguas superficiales del río Portoviejo se presentan en la tabla 5. No se encontró ningún contaminante en concentraciones cuantificables a niveles de $\mu \mathrm{g} / \mathrm{mL}$ en ninguna de las muestras analizadas, esto se puede justificar por ciertos factores demográficos, como el tamaño poblacional de las principales ciudades ubicadas a orillas del río Portoviejo; lo cual influye significativamente en el aporte per cápita de contaminantes emergentes a los cuerpos de agua superficial (Gavrilescu et al., 2015). En una investigación similar realizada por Campanha et al. (2015), en el río Monjolinho, São Paulo - Brasil, donde la población bordea alrededor de los 12,33 millones de habitantes, las concentraciones más bajas en las muestras de agua superficial fueron para el diclofenaco sódico $(0,04$ $\mathrm{ng} / \mathrm{L})$ y el naproxeno $(0,1 \mathrm{ng} / \mathrm{L})$.

Además en un estudio realizado a nivel local, (Voloshenko-Rossin et al., 2015) analizaron muestras de agua de los ríos San Pedro, Guayllabamba y Esmeraldas, los cuales abastecen de líquido vital a la provincia de Esmeraldas y en su trayecto reciben las aguas residuales del Distrito Metropolitano de Quito. Aquí se encontraron concentraciones de fármacos como cafeína, sulfametoxazol y venlafaxina por encima de los 2000 $\mathrm{ng} / \mathrm{L}$ pero no fue posible detectar otro tipo de fármacos en el rango de ng/L. 
Tabla 5. Antiinflamatorios no esteroideos en muestras de agua superficial.

\begin{tabular}{lccccccc}
\hline \multirow{2}{*}{ Fármacos } & \multirow{2}{*}{ Unidades } & \multicolumn{7}{l}{ Puntos de Muestreo } & & & & \\
\cline { 3 - 8 } & & P1 & P2 & P3 & P4 & P5 & P6 \\
\hline ASP & $\mu \mathrm{g} / \mathrm{mL}$ & nd & $<\mathrm{LOQ}$ & nd & $<\mathrm{LOQ}$ & nd & $<\mathrm{LOQ}$ \\
$\mathrm{KTL}$ & $\mu \mathrm{g} / \mathrm{mL}$ & nd & nd & nd & nd & nd & nd \\
$\mathrm{MLX}$ & $\mu \mathrm{g} / \mathrm{mL}$ & nd & nd & nd & nd & nd & nd \\
NPX & $\mu \mathrm{g} / \mathrm{mL}$ & nd & nd & nd & nd & nd & $<\mathrm{LOQ}$ \\
DCF & $\mu \mathrm{g} / \mathrm{mL}$ & nd & nd & nd & nd & nd & nd \\
IBF & $\mu \mathrm{g} / \mathrm{mL}$ & nd & nd & nd & nd & nd & nd \\
\hline
\end{tabular}

${ }^{*}$ nd $=$ No detectable

* $\mathrm{LOQ}=$ Límite de cuantificación

Fuente: Elaboración propia.

\section{Muestras de agua potable}

La tabla 6 muestra las concentraciones de los compuestos objeto de estudio en muestras de agua potable, las cuales fueron recolectadas en puntos de muestreo de tres cantones diferentes de la provincia de Manabí, cuya principal fuente de agua es el río Portoviejo. No se encontró ningún contaminante emergente en concentraciones cuantificables a niveles de $\mu \mathrm{g} / \mathrm{mL}$ en ninguna de las muestras analizadas, $y$ aunque no se pudo cuantificar ningún analito de interés es importante señalar que normalmente solo se han determinado niveles extremadamente bajos de fármacos en muestras de agua potable de todo el mundo (Muñoz et al., 2008). En un estudio anterior realizado en la región, Sodré et al. (2010) analizaron muestras de agua potable de la ciudad las Campinas, en Brasil y pudieron detectar seis contaminantes de los cuales la cafeína $(0,22 \pm 0,06 \mu \mathrm{g} / \mathrm{L})$ y el bisfenol A $(0,16 \pm 0,03 \mu \mathrm{g} / \mathrm{L})$ presentaron las concentraciones más alta.

Tabla 7. Antiinflamatorios no esteroideos en muestras de agua potable.

\begin{tabular}{lcccc}
\hline \multirow{2}{*}{ Fármacos } & \multirow{2}{*}{ Unidades } & Puntos de Muestreo & & \\
\cline { 3 - 5 } & $\mu \mathrm{g} / \mathrm{mL}$ & Manta & Portoviejo & Rocafuerte \\
\hline ASP & $\mu \mathrm{g} / \mathrm{mL}$ & nd & nd & nd \\
$\mathrm{KTL}$ & $\mu \mathrm{g} / \mathrm{mL}$ & nd & nd & nd \\
$\mathrm{MLX}$ & $\mu \mathrm{g} / \mathrm{mL}$ & nd & nd & nd \\
NPX & $\mu \mathrm{g} / \mathrm{mL}$ & nd & nd & nd \\
DCF & $\mu \mathrm{g} / \mathrm{mL}$ & nd & nd & nd \\
IBF & & &
\end{tabular}

Fuente: Elaboración propia. 


\section{Conclusiones}

La técnica analítica desarrollada para la determinación de antinflamatorios no esteroideos mediante cromatografía líquida de alta eficacia con un detector ultravioleta - visible multiespectral de arreglo de diodos, permitió la determinación simultánea de los seis fármacos estudiados en muestras de agua para consumo. Así mismo de acuerdo al procedimiento de validación realizado el método presenta confiabilidad respecto a la cuantificación de contaminantes emergentes de baja polaridad.

La evaluación del impacto de las descargas de efluentes residuales urbanos, hospitalarios y centros médicos sobre el río Portoviejo muestra que, aunque dicha influencia ciertamente existe y varios contaminantes emergentes persisten, todos ellos se encuentran en niveles significativamente bajos y no es posible detectarlos a niveles de concentración en el orden de los $\mu \mathrm{g} / \mathrm{mL}$.

Ciertos factores demográficos, como el tamaño de la población, pudieron haber incidido en el resultado de la investigación, ya que el aporte per cápita de contaminantes fue poco significativo en comparación con otras ciudades del mundo.

Considerando que estos compuestos se encuentran diseminados en el ambiente y aún no están regulados por la legislación ambiental ecuatoriana, los resultados reportados pueden ayudar a componer una base de datos para el monitoreo $y$ seguimiento de contaminantes emergentes en cuerpos de agua superficiales, lo cual es extremadamente necesario para garantizar la salud de la población y una gestión sostenible de los recursos hídricos en el contexto local.

\section{Recomendaciones}

Se recomienda desarrollar técnicas analíticas más sensibles para la determinación de fármacos en muestras acuosas a niveles de ng/L. 


\section{Bibliografía}

Campanha, M. B., Awan, A. T., de Sousa, D. N. R., Grosseli, G. M., Mozeto, A. A., \& Fadini, P. S. (2015). A 3-year study on occurrence of emerging contaminants in an urban stream of São Paulo State of Southeast Brazil. Environmental Science and Pollution Research, 22(10), 7936-7947.

https://doi.org/10.1007/s1135 6-014-3929-x

Ebele, A. J., Abou-Elwafa Abdallah, M., \& Harrad, S. (2017). Pharmaceuticals and personal care products (PPCPs) in the freshwater aquatic environment. Emerging Contaminants, 3(1), 1-16.

https://doi.org/10.1016/j.emco n.2016.12.004

Farré, M. la, Pérez, S., Kantiani, L., \& Barceló, D. (2008). Fate and toxicity of emerging pollutants, their metabolites and transformation products in the aquatic environment. TrAC - Trends in Analytical Chemistry, 27(11), 991-1007. https://doi.org/10.1016/j.trac.2 008.09.010

Fatta, D., Achilleos, A., Nikolaou, A., \& Meriç, S. (2007). Analytical methods for tracing pharmaceutical residues in water and wastewater. TrAC Trends in Analytical
Chemistry, 26(6), 515-533. https://doi.org/10.1016/j.trac.2 007.02.001

Fatta-Kassinos, D., Kalavrouziotis, I. K., Koukoulakis, P. H., \& Vasquez, M. I. (2011). The risks associated with wastewater reuse and xenobiotics in the agroecological environment. Science of the Total Environment, 409(19), 35553563.

https://doi.org/10.1016/j.scitot env.2010.03.036

Gavrilescu, M., Demnerová, K., Aamand, J., Agathos, S., \& Fava, F. (2015). Emerging pollutants in the environment: Present and future challenges in biomonitoring, ecological risks and bioremediation. New Biotechnology, 32(1), 147156.

https://doi.org/10.1016/j.nbt.2 014.01.001

Geissen, V., Mol, H., Klumpp, E., Umlauf, G., Nadal, M., van der Ploeg, M., van de Zee, S. E. A. T. M., \& Ritsema, C. J. (2015). Emerging pollutants in the environment: A challenge for water resource management. International Soil and Water Conservation Research, 3(1), 57-65. https://doi.org/10.1016/j.iswcr .2015.03.002

Houtman, C. J. (2010). Emerging contaminants in surface waters and their relevance for 
the production of drinking water in Europe. Journal of Integrative Environmental Sciences, 7(4), 271-295. https://doi.org/10.1080/19438 15X.2010.511648

Jardim, W. F., Montagner, C. C., Pescara, I. C., Umbuzeiro, G. A., di Dea Bergamasco, A. M., Eldridge, M. L., \& Sodré, F. F. (2012). An integrated approach to evaluate emerging contaminants in drinking water. Separation and Purification Technology, 84 , 3-8. https://doi.org/10.1016/j.sepp ur.2011.06.020

Jekel, M., Ruhl, A. S., Meinel, F., Zietzschmann, F., Lima, S. P., Baur, N., Wenzel, M., Gnirß, R., Sperlich, A., Dünnbier, U., Böckelmann, U., Hummelt, D., van Baar, P., Wode, F., Petersohn, D., Grummt, T., Eckhardt, A., Schulz, W., Heermann, A., ... Mutz, D. (2013).

Anthropogenic organic micro-pollutants and pathogens in the urban water cycle: Assessment, barriers and risk communication (ASKURIS). Environmental Sciences Europe, 25(1), 1-8. https://doi.org/10.1186/21904715-25-20

Jensen, O., \& Wu, H. (2018). Urban water security indicators: Development and pilot. Environmental Science and Policy, 83(September 2017),
33-45.

https://doi.org/10.1016/j.envs ci.2018.02.003

Kostopoulou, M., \& Nikolaou, A. (2008). Analytical problems and the need for sample preparation in the determination of pharmaceuticals and their metabolites in aqueous environmental matrices. TrAC - Trends in Analytical Chemistry, 27(11), 10231035.

https://doi.org/10.1016/j.trac.2 008.09.011

Larsen, T. A., \& Gujer, W. (1997). The concept of sustainable urban water management. Water Science and Technology, 35(9), 3-10. https://doi.org/10.1016/S0273 -1223(97)00179-0

Leiva, D. (2019). Evaluación del desempeño de un método analítico para la determinación de fármacos en agua de consumo por cromatografía de líquidos con detección ultravioleta (HPLCUV). In Repositorio de la Pontificia Universidad Católica del Ecuador (Vol. 1, Issue http://repositorio.puce.edu.ec/ handle/22000/17400

Lin, A. Y. C., Wang, X. H., \& Lin, C. F. (2010). Impact of wastewaters and hospital effluents on the occurrence of controlled substances in 
surface

waters.

Chemosphere, 81(5), 562-

570.

https://doi.org/10.1016/j.chem

osphere.2010.08.051

Marsalek, J. (2014). Urban Water Cycle Processes and Interactions. In Urban Water Cycle Processes and Interactions. CRC Press. https://doi.org/10.1201/97814 82288544

Muñoz, I., José Gómez, M., MolinaDíaz, A., Huijbregts, M. A. J., Fernández-Alba, A. R., \& García-Calvo, E. (2008). Ranking potential impacts of priority and emerging pollutants in urban wastewater through life cycle impact assessment. Chemosphere, 74(1), 37-44. https://doi.org/10.1016/j.chem osphere.2008.09.029

Nikolaou,

A.

(2013).

Pharmaceuticals and related compounds as emerging pollutants in water: Analytical aspects. Global Nest Journal, 15(1), $1-12$. https://doi.org/10.30955/gnj.0 00969

Nikolaou, A., Meric, S., \& Fatta, D. (2007). Occurrence patterns of pharmaceuticals in water and wastewater environments. Analytical and Bioanalytical Chemistry, 387(4), 1225-1234. https://doi.org/10.1007/s0021 6-006-1035-8
Ortiz-prado, E., Galarza, C., Cornejo, F., \& Ponce, J. (2014). Acceso a medicamentos y situación del mercado farmacéutico en Ecuador. 36(1).

Pal, A., He, Y., Jekel, M., Reinhard, M., \& Gin, K. Y. H. (2014). Emerging contaminants of public health significance as water quality indicator compounds in the urban water cycle. Environment International, 71, 46-62. https://doi.org/10.1016/j.envin t.2014.05.025

Patel, P. N., Samanthula, G., Shrigod, V., Modh, S. C., \& Chaudhari, J. R. (2013). RPHPLC Method for Determination of Several NSAIDs and Their Combination Drugs. Chromatography Research International, 2013, 1-13. https://doi.org/10.1155/2013/ 242868

Patrolecco, L., Ademollo, N., Grenni, P., Tolomei, A., Barra Caracciolo, A., \& Capri, S. (2013). Simultaneous determination of human pharmaceuticals in water samples by solid phase extraction and HPLC with UVfluorescence detection. Microchemical Journal, 107, 165-171.

https://doi.org/10.1016/j.micro c. 2012.05 .035

Peña-Guzmán, C., Ulloa-Sánchez, S., Mora, K., Helena-Bustos, 
R., Lopez-Barrera, E., Alvarez, J., \& RodriguezPinzón, M. (2019). Emerging pollutants in the urban water cycle in Latin America: A review of the current literature. Journal of Environmental Management, 237(February), 408-423.

https://doi.org/10.1016/j.jenv man.2019.02.100

Rivera-Utrilla, J., Sánchez-Polo, M., Ferro-García, M. Á., PradosJoya, G., \& Ocampo-Pérez, R. (2013). Pharmaceuticals as emerging contaminants and their removal from water. A review. Chemosphere, 93(7), 1268-1287.

https://doi.org/10.1016/j.chem osphere.2013.07.059

Rosas, J., Santos, G., Martín, R., Cortés, R., \& Álvarez, A. (2013). Capítulo 26: Antiinflamatorios no esteroideos. In Enfermedades Reumáticas. Actualización SVR (Issue 1, pp. 469-476).

Ruhí, A., Acuña, V., Barceló, D., Huerta, B., Mor, J. R., Rodríguez-Mozaz, S., \& Sabater, S. (2016). Bioaccumulation and trophic magnification of pharmaceuticals and endocrine disruptors in a Mediterranean river food web. Science of the Total Environment, 540, 250-259. https://doi.org/10.1016/j.scitot env.2015.06.009
Singh, K.B., Waikar, S.B., and Padmane, S.P. (2012). A Validated RP-HPLC Method for the Simultaneous Estimation of Paracetamol and Naproxen in Tablet Formulation. International Journal of Pharmaceutical Sciences and Research, 3(11), 4270-4275.

Sodré, F. F., Locatelli, M. A. F., \& Jardim, W. F. (2010). Occurrence of emerging contaminants in Brazilian drinking waters: A sewage-totap issue. Water, Air, and Soil Pollution, 206(1-4), 57-67. https://doi.org/10.1007/s1127 0-009-0086-9

Voloshenko-Rossin, A., Gasser, G., Cohen, K., Gun, J., CumbalFlores, L., Parra-Morales, W., Sarabia, F., Ojeda, F., \& Lev, O. (2015). Emerging pollutants in the Esmeraldas watershed in Ecuador: Discharge and attenuation of emerging organic pollutants along the San PedroGuayllabamba - Esmeraldas rivers. Environmental Sciences: Processes and Impacts, 17(1), 41-53. https://doi.org/10.1039/c4em0 0394b 